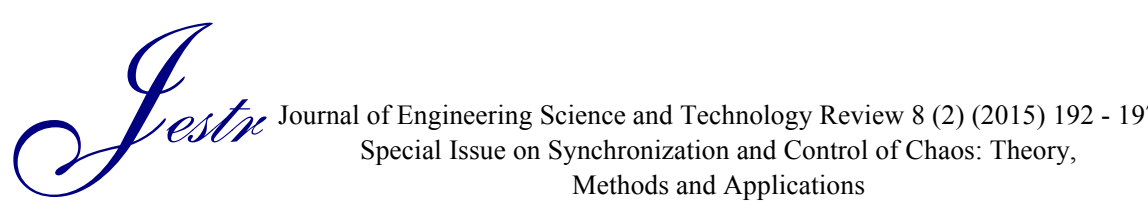

Research Article

\title{
Chaos Control in Memristor-based Oscillators Using Intelligent Sliding Mode Control
}

\author{
Amir Hossein Abolmasoumi ${ }^{*}{ }^{1}$ and Somayeh Khosravinejad ${ }^{2}$ \\ ${ }^{I}$ Department of Electrical engineering, Faculty of Engineering, Arak University, Arak 38156-8- 8349, Iran. \\ ${ }^{2}$ Department of Electrical engineering, Arak Branch, Islamic Azad University, Iran.
}

\begin{abstract}
In this paper, Intelligent Sliding Mode Control of chaos in a memristor- based Chua's oscillator is investigated. In order to gain stabilization and tracking of a sinusoidal input, an appropriate sliding surface is proposed and sliding gain is tuned. Also, to avoid the chattering phenomenon in traditional sliding mode controller, and to reduce the hitting time of the controlled system, an especial genetic algorithm optimization method is suggested. By defining a new objective function and searching for optimal the controller parameters the convergence time and chattering are reduced considerably. The usefulness of the proposed controller with intelligent tuning method for chaos control of memristorbased oscillators is demonstrated in memristor- based Chua's circuit.
\end{abstract}

Keywords: Chaos Control, Memristor, Sliding Mode Control, Genetic Algorithm.

\section{Introduction}

Chaotic behavior may occur in many mechanical and electronic oscillators, in chemical reactions, in laser cavities, etc. Various methods are used to control chaos such as linear Backstepping control [1, 2], feedback control [3, 4], impulsive control [5] and adaptive control [6].

As a fourth circuit element, memristors, were proposed by Leon Chua in 1971 [7]. A team at Hewlett-Packard Labs announced the fabrication of a nanometer-size solid-state two-terminal device called the memristor in science on May, 2008 [8], by titanium dioxide thin films. This passive electronic element represents some properties of resistors and has the same measurement unit (ohms). The main difference between memristors and ordinary resistor is that memristors have the function of remembering the history state as implied by its name; a contraction of memory resistor [9].

Oscillators are the prime signal generators. Electronic oscillators are electrical circuits that produce sine or square wave in their output. Memristor based oscillators often are achieved by replacing one or more element of traditional oscillator circuits with a memristive system.

For example, memristor based Chua's Oscillator is evolved from Chua's chaotic circuit by replacing the Chua's diode with a two terminal circuit consisting of a negative conductance and a flux-controlled memristor [10]. So far,

* E-mail address: a-abolmasoumi@araku.ac.ir ISSN: 1791-2377 (C) 2015 Kavala Institute of Technology. All rights reserved. investigators have proposed several memristor- based oscillator [11-14]. In [15-19], various methods have been used to control the chaos in the chaotic oscillators and memristor- based chaotic oscillators.

Variable structure control with sliding mode is a nonlinear control strategy which is used in nonlinear problems to achieve the goals such as stabilization, tracking the reference input and controlling systems with uncertainties. A standard sliding mode controller design consists of two phases. The first phase, which is called the reaching mode, is when the initial state trajectory of the system is approaching to the arbitrary sliding surface (chosen by the designer).In the second phase, which is called the sliding mode, the system state after reaching the desired surface, it starts sliding on the surface and stay on that. Chattering phenomenon will occur after the first time system state hits the sliding surface [20].

Traditional Sliding Mode Control (SMC) always suffers from two important issues, convergence time and chattering phenomenon. If the system states reach the sliding surface in limited time, it could be shown that the system stability and the desired performance will be guaranteed. Chattering and hitting time reduction in SMC would be obtained by choosing the optimal controller parameters using intelligent optimization methods such as Genetic Algorithm (GA), Particle Swarm Optimization (PSO) and Bee Colony [21-25]. In [22], GA is applied to search the optimal controller parameters including switching function coefficient, switching gain adaptive coefficient, sigmoid function coefficient and observer coefficient for chattering reduction but hitting time reduction is not considered. In 
[23], the sliding mode parameters have been obtained by using GA optimization technique, but [23] did not mention to reduction the chattering and to shorten the hitting time. In [25], a genetic-based sliding mode fuzzy controller is developed to obtain width of boundary layer and control gain for chattering reduction and hitting time shortening, but the effect of optimization of sliding surface coefficients on chattering and hitting time reduction is not considered.

In this paper, we shall consider the intelligent sliding mode control of a memristor- based oscillators. In the memristor- based oscillators, nonlinear memristor may be the occurrence of chaos in oscillator. The Chua's circuit is an extensively studied and well-understood chaotic system. Chua's circuit exhibits complex nonlinear dynamics such as bifurcations and chaos. Where, Chaos control in memristorbased Chua's oscillator is performed using intelligent sliding mode control to stabilization and tracking of a sinusoidal input. We consider the stability of controller using the Lyapunov criterion. Furthermore, GA is applied to determine the controller parameters to eliminate chattering problem and to shorten hitting time in SMC.

\section{Traditional Sliding Mode Control}

In traditional SMC, dynamic characteristic of the system are used to define the sliding surface. Commonly, the sliding surface is a linear hyperplane of system states. The sliding surface is proposed in [20] as:

$$
S(t)=\left(\frac{d}{d t}+\lambda\right)^{n-1} e(t)
$$

where $n$ is the order of system and $\lambda$ is a strictly positive constant which can be chosen by designer arbitrarily, taken to be the bandwidth of the system. $e(t)$ is the tracking error, which is defined as follows:

$e(t)=y_{\text {out }}(t)-y_{d}$

where $y_{d}$ is the desired output. To ensure that the sliding mode exists on the switching surface, the following condition must be satisfied [20]:

$$
\frac{1}{2} \frac{d}{d t} s^{2} \leq-\eta|s|
$$

where $\eta>0$ is a constant.

Now, consider the 2-order system in a canonical model of state as follows:

$$
\begin{aligned}
& \left\{\begin{array}{l}
\dot{x}_{1}=x_{2} \\
\dot{x}_{2}=f(\mathbf{x})+b(\mathbf{x}) \mathrm{u}+\mathrm{d}(\mathrm{t})
\end{array}\right. \\
& y_{\text {out }}=x_{1}
\end{aligned}
$$

where $y_{\text {out }}$ is the system output, $\mathbf{x}=\left[x_{1}(t), x_{2}(t)\right]$ is the states vector, $u(t)$ is the control input, $f(\mathbf{x})$ and $b(\mathbf{x})$ are nonlinear functions and $d(t)$ is external disturbance and parameters uncertainty which is defined as $|d(t)| \leq L$. From Eq. (1), we define a sliding surface for the system (4) as follows:

$$
S(t)=\dot{x}_{1}+\lambda x_{1}=x_{2}+\lambda x_{1}
$$

Dynamic in sliding mode is obtained as:

$$
\dot{S}=\dot{x}_{2}+\lambda \dot{x}_{1}=f(\mathbf{x})+b(\mathbf{x}) \mathrm{u}+\mathrm{d}(\mathrm{t})+\lambda x_{2}=0
$$

A conventional control law that satisfies Eq. (3) is defined as:

$u=u_{e q}-k \operatorname{sgn}(S), \mathrm{k}>L+\eta$

where sgn is sign function and $u_{e q}$ is equivalent control which can be obtained from Eq. (6):

$\mathrm{u}_{\mathrm{eq}}=\frac{-f(\mathbf{x})-\mathrm{d}(\mathrm{t})-\lambda x_{2}}{b(\mathbf{x})}$

According to the Lyapunov theorem, we can choose a Lyapunov function candidate as:

$V=\frac{1}{2} S^{2}$

$\dot{V}=S \dot{S}=S\left[f(\mathbf{x})+b(\mathbf{x}) \mathrm{u}+\mathrm{d}(\mathrm{t})+\lambda x_{2}\right]$

Substituting Eq. (7) and Eq. (8) into Eq. (10), we have

$\dot{V}=S(-\operatorname{ksgn}(\mathrm{S}))=-\mathrm{k}|S| \leq 0$,

Chattering phenomenon will occur after the first time state hits the sliding surface. Chattering must be reduced or eliminated, this can be achieved by smoothing the control discontinuity in a thin boundary layer, $\phi$, neighboring the switching surface. The modified control low can be described as follows:

$u=u_{e q}-k \operatorname{sat}\left(\frac{S}{\phi}\right), \phi>0$

\section{Intelligent SMC Based on Genetic Algorithm}

Genetic algorithms (GA) are stochastic global search methods that simulate natural biological evolution. GAs stems from a population of potential solution estimates along with applying the principle of survival of the fittest to produce more appropriate approximations to a solution in each stage. Genetic algorithms belong to the larger class of evolutionary algorithm (EA), which generate solutions to optimization problems using techniques, such as inheritance, mutation, selection, and crossover. GAs use fitness function information instead of derivatives or other auxiliary knowledge. In GA, we need to choose some relative parameters, such as generations, population size, crossover rate, mutation rate, and coding length of chromosome [26].

On the other hand, it is obvious that the convergence rate of SMC can be controlled by appropriate choice of coefficients $c_{1}, c_{2}, \mathrm{~K}, c_{n-1}$ and $k$, also the width of boundary layer $\phi$ affects the chattering amplitude of the sliding mode controller. Therefore, selecting the appropriate 
values of these parameters using genetic algorithms can improve the performance of sliding mode controller. We can define parameters $k, C=\left[c_{1}, c_{2}, \mathrm{~K}, c_{n-1}, c_{n}\right]$ and $\phi$ as a set of parameters $\left(R_{i}=(\mathrm{C}, k, \phi)\right)$ that are used to form a chromosome in genetic algorithms. We use the hitting time and chattering of the controlled system as the performance index of fitness function. Fitness function is defined as follows:

$$
F\left(R_{i}\right)=\int S d t+\left(u-u_{e q}\right)^{2}
$$

The proposed fitness function can choose the controller parameters so that the hitting time and chattering phenomenon are reduced. The first part of the proposed fitness function (12) can provide the smaller chattering magnitude and the shorter hitting time by minimizing the area under the curve of the sliding surface $S$, (as a result of optimizing of the coefficients $\left.C=\left[c_{1}, c_{2}, \mathrm{~K}, c_{n-1}, c_{n}\right]\right)$. The second term of the fitness function will reduce the amplitude of the chattering by optimizing the width of boundary layer $\phi$, and also will shorten the hitting time by optimizing the parameter value $k$.

\section{Memristor Based Chua's Oscillator}

Chua's oscillator circuit is known as a primary source for studying and generating chaos in electronic circuits. Chua's circuit consists of a linear inductor, two capacitors, a linear resistor and nonlinear Chua's diode [27]. Memristor-based Chua's oscillator is evolved from Chua's chaotic circuit by replacing the Chua's diode with a two terminal circuit consisting of a negative conductance and a flux-controlled memristor, Fig.1 [10].

Flux-controlled memristor described by the function $W(\phi)$, which is called memristance, is defined by the equations:

$W(\phi)=d q(\phi) / d \phi$

where $q(\phi)$ is a smooth continuous cubic monotoneincreasing nonlinearity function as follows:

$$
q(\phi)=a \phi+b \phi^{3}
$$

Consequently, the memristance $W(\phi)$ is defined by the equation $W(\phi)=a+3 \phi^{2}$. Thus, the relationship between the voltage and the current through the memristor is given by:

$$
i(t)=W(\phi) \cdot \mathrm{v}(\mathrm{t})
$$

From Fig. 1, we can obtain a set of four first-order differential equations, which are used to define the relationship among the four circuit variables $\left(v_{1}, v_{2}, i_{3}, \phi\right)$ :

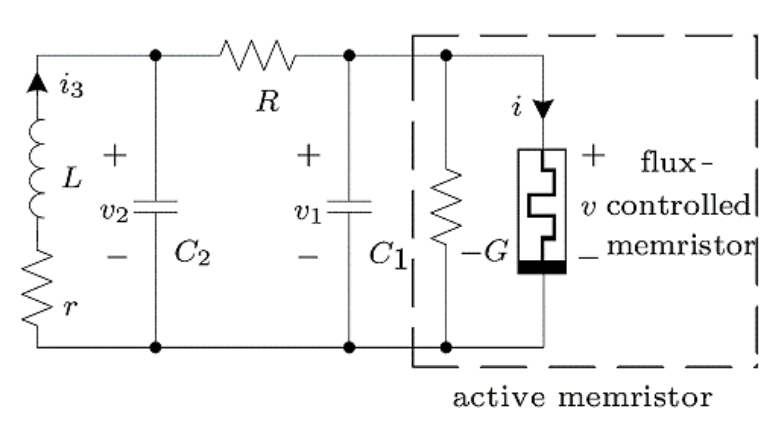

Fig. 1. Memristor-based Chua's oscillator.

$$
\left\{\begin{array}{l}
\frac{d v_{1}}{d t}=\frac{1}{R C_{1}}\left[v_{2}-v_{1}+G R v_{1}-R W(\phi) v_{1}\right] \\
\frac{d v_{2}}{d t}=\frac{1}{R C_{2}}\left[v_{1}-v_{2}+R i_{3}\right] \\
\frac{d i_{3}}{d t}=-\frac{1}{L} v_{2}-\frac{r}{L} i_{3} \\
\frac{d \phi}{d t}=v_{1}
\end{array}\right.
$$

To simplify the equations, variables and parameters can be changed as follows:

$$
\begin{aligned}
& x_{1}=\phi, x_{2}=v_{1}, x_{3}=\mathrm{v}_{2}, \mathrm{x}_{4}=i_{3}, \alpha=1 / C_{1}, \beta=1 / L, \gamma=r / L, \\
& \xi=G, C_{2}=1 \text { and } R=1 .
\end{aligned}
$$

With these variable changes, the state equations are transformed into the form below:

$\left\{\begin{array}{l}\dot{x}_{1}=x_{2} \\ \dot{x}_{2}=\alpha\left(x_{3}-x_{2}+\xi x_{2}-W\left(x_{1}\right) x_{2}\right) \\ \dot{x}_{3}=x_{2}-x_{3}+x_{4} \\ \dot{x}_{4}=-\left(\beta x_{3}+\gamma x_{4}\right)\end{array}\right.$

The system (17) can be expressed in the canonical form using linearization transformation of input state. For the linearization transformation of input state, at the first step we write the state system (17) as follows:

$$
\begin{aligned}
& \left\{\begin{array}{l}
\dot{x}_{1}=x_{2} \\
\dot{x}_{2}=\alpha\left(x_{3}-x_{2}+\xi x_{2}-W\left(x_{1}\right) x_{2}\right) \\
\dot{x}_{3}=x_{2}-x_{3}+x_{4} \\
\dot{x}_{4}=-\left(\beta x_{3}+\gamma x_{4}\right)+\mathrm{u}
\end{array}\right. \\
& y_{\text {out }}=x_{1}
\end{aligned}
$$

After applying linearization transformation of the input state for the system (18), we have

$$
\left\{\begin{array}{l}
\dot{z}_{1}=z_{2} \\
\dot{z}_{2}=z_{3} \\
\dot{z}_{3}=z_{4} \\
\dot{z}_{4}=v=f(\mathbf{x})+g(\mathbf{x}) u
\end{array}\right.
$$

where 


$$
\begin{aligned}
f(\mathbf{x})= & -6 \alpha b x_{2}^{3}-12 \alpha^{2} b x_{1} x_{2}\left(x_{3}-x_{2}+\xi x_{2}-W\left(x_{1}\right) x_{2}\right) \\
& +\alpha^{2}\left(-1+\xi-W\left(x_{1}\right)\right)\left[x_{2}-x_{3}+x_{4}\right. \\
& -\alpha\left(-1+\xi-W\left(x_{1}\right)\right)\left(x_{3}-x_{2}+\xi x_{2}-W\left(x_{1}\right) x_{2}\right) \\
& \left.-6 b x_{1} x_{2}^{2}\right]+\alpha^{2}\left(-6 b x_{1} x_{2}\right)\left(x_{3}-x_{2}+\xi x_{2}-W\left(x_{1}\right) x_{2}\right) \\
& +\alpha^{2}\left(x_{3}-x_{2}+\xi x_{2}-W\left(x_{1}\right) x_{2}\right)-\alpha\left(x_{2}-x_{3}+x_{4}\right)+\alpha\left(-\beta x_{3}-\gamma x_{4}\right)
\end{aligned}
$$

$g(\mathbf{x})=\alpha$

\section{Traditional SMC for Chua's Memristor-based Oscillator}

In this section, we design SMC method for chaos control in the Memristor-based Chua's oscillator for two main goals: 1-stabilization, 2-tracking the sinusoidal input. Here, neglect the effect of the external disturbance and parameter uncertainties i.e. $d(t)=0$.

We have for system (19), by considering tracking error as:

$\left\{\begin{array}{l}e_{1}=z_{1}-y_{d} \\ e_{2}=z_{2}-\dot{y}_{d} \\ e_{3}=z_{3}-\ddot{y}_{d} \\ e_{4}=z_{4}-y_{d}^{(3)}\end{array} \Rightarrow\left\{\begin{array}{l}\dot{e}_{1}=e_{2} \\ \dot{e}_{2}=e_{3} \\ \dot{e}_{3}=e_{4} \\ \dot{e}_{4}=f(\mathbf{x})+b(\mathbf{x}) u-y_{d}^{(4)}\end{array}\right.\right.$

In the stabilization and tracking problems, $y_{d}$ is taken to be zero and $\sin (t)$, respectively.

According to Eq.1, we define a sliding surface as follows:

$$
\begin{aligned}
S & =\left(\frac{d}{d t}+\lambda\right)^{3} e(t) \\
& =c_{1} e_{1}+c_{2} e_{2}+c_{3} e_{3}+c_{4} e_{4}
\end{aligned}
$$

where $C=\left[c_{1}, c_{2}, c_{3}, c_{4}\right]$ are positive constant and that can determine by $\lambda$ value $\left(c_{1}=\lambda^{3}, c_{2}=3 \lambda^{2}, c_{3}=3 \lambda\right.$ and $\left.\mathrm{c}_{4}=1\right)$. We can obtain the equivalent control law from $\dot{S}=0$ as:

$u_{e q}=\frac{-c_{1} e_{2}-c_{2} e_{3}-c_{3} e_{4}-f(\mathbf{x})+\mathrm{y}_{d}{ }^{(4)}}{b(\mathbf{x})}$

\section{Simulation Result}

In this section, the effectiveness of the proposed methods is validated by simulation and the simulation resulted from traditional SMC and GA-SMC are compared. Threshold value of saturation function is set equal to 0.02 . In GA's parameter setting, we set the population size equal to 50 , the number of generations is selected to be 100 , the crossover rate is chosen as 0.8 and mutation rate is 0.04 . Memristorbased Chua's oscillator parameter values are considered as follows:

$\alpha=9.8, \quad \beta=100 / 7, \quad \gamma=0, \quad \xi=9 / 7, a=-0.667 * 10^{-3}$ and $b=0.029 * 10^{-3}$.
For the initial conditions $\left(10^{-3}, 10^{-3}, 10^{-10}, 10^{-4}\right)$, the oscillator shows chaotic behavior (Fig. 2) and displays a symmetrical 2-scroll chaotic attractor as shown in Fig. 3.
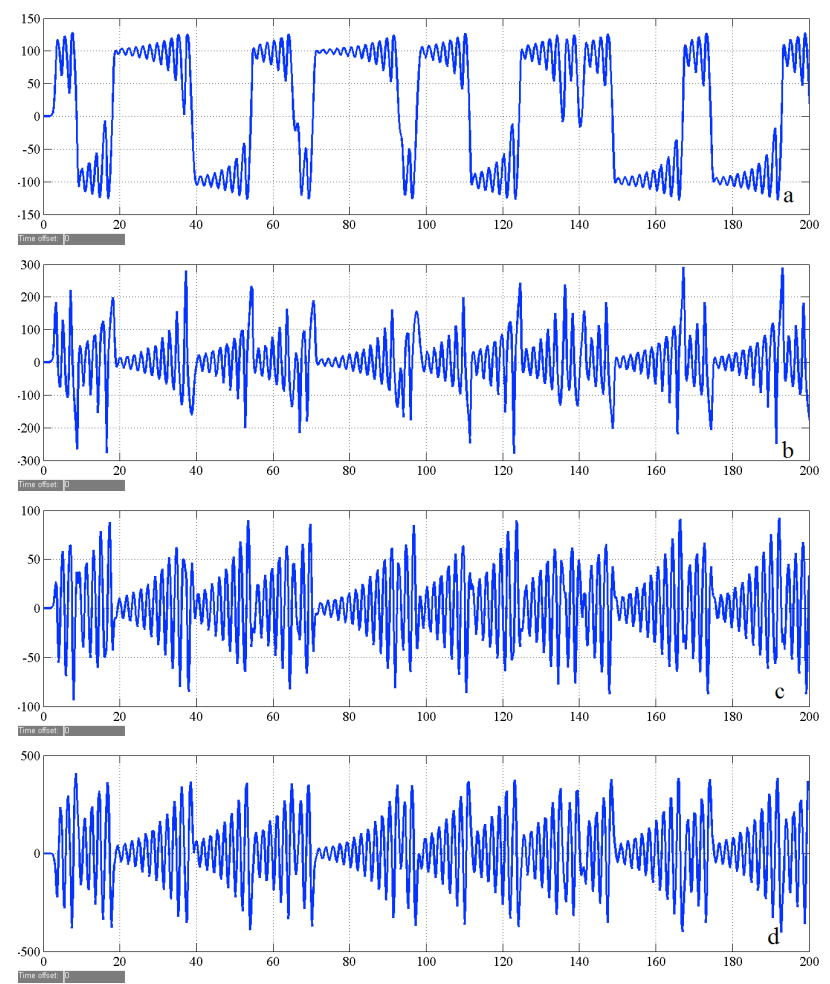

Fig. 2. Plots of states of system (17) for initial conditions $\left(10^{-3}, 10^{-3}, 10^{-10}, 10^{-4}\right)$, (a) $x_{1}$, (b) $x_{2}$, (c) $x_{3}$ and (d) $x_{4}$.
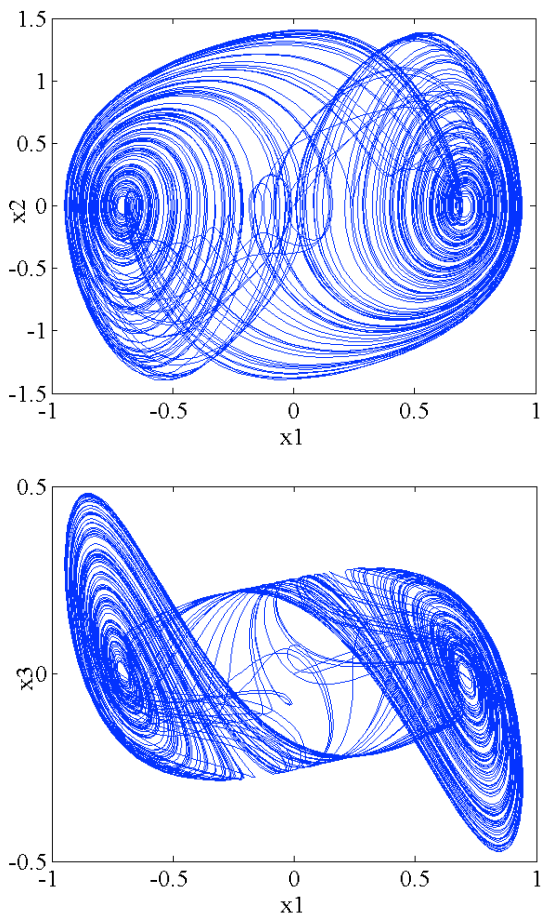

Fig. 3. Chaotic attractor of Memristor- based Chua's oscillator.

In Traditional SMC, parameter values can be selected by experience to achieve appropriate convergence rate and the low magnitude of chattering. 
In Eq. 20, by choosing $\lambda=1.1$, values of $\mathrm{C}$ parameters are obtained as follows:

\section{$\lambda=1.1 \Rightarrow c_{1}=1.33, c_{2}=3.63, c_{3}=3.3$.}

Due to the influence of the parameters $k$ and $\phi$ on the convergence time and chattering magnitude, we have chosen the values of $k$ and $\phi$ for an appropriate convergence and low chattering magnitude, as $k=8.5$ and $\phi=5$ by experience.

The selected values of parameters are used for the both stabilization and tracking problems.

In GA-based intelligent SMC, the values of parameters are optimized as follows:

In stabilization problem:

$c_{1}=3.766, c_{2}=6.079, c_{3}=3.382, c_{4}=1.054, k=1.1$ and $\phi=0.854$.

In tracking problem:

$c_{1}=0.392, c_{2}=0.79, c_{3}=0.214, c_{4}=0.026, k=3.175$, and $\phi=0.579$.

The simulation results of stabilization of system states using GA-based intelligent SMC and SMC methods are shown in Fig. 4. Fig. 5 shows the time response of the surface $\mathrm{S}$ and control $\mathrm{u}$ in this simulation.

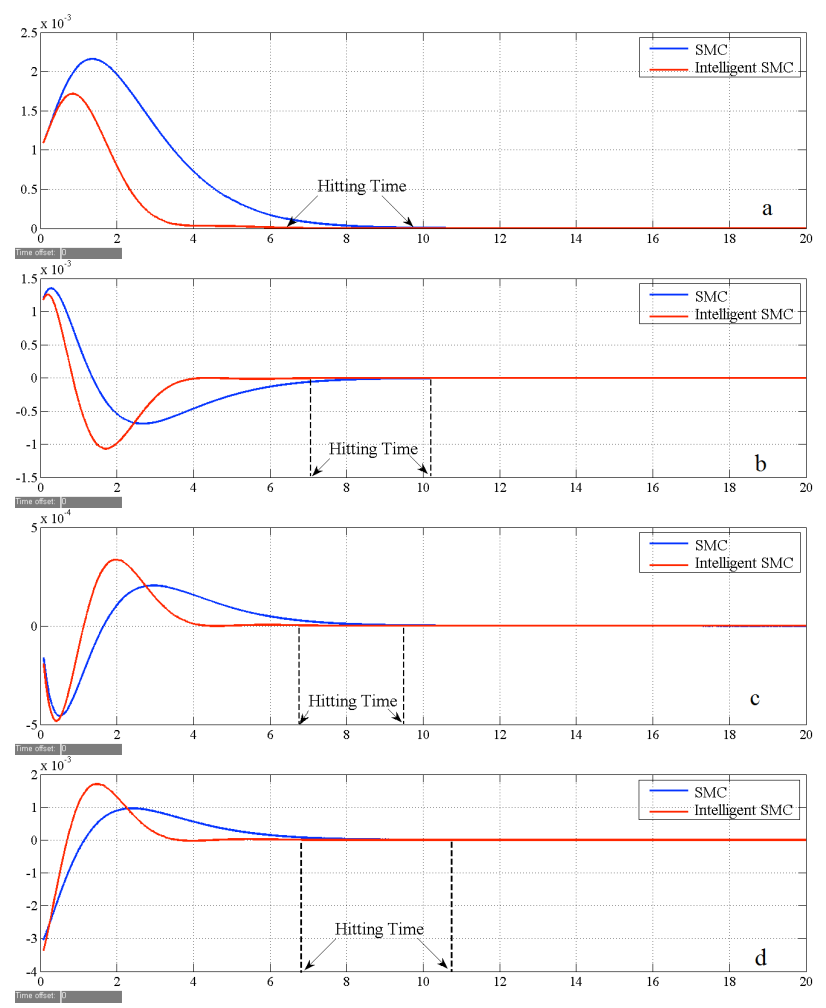

Fig. 4. The simulation results of stabilization of system states using GA-SMC and SMC, (a) $x_{1}$, (b) $x_{2}$, (c) $x_{3}$ and (d) $x_{4}$.

As shown in Fig. 4, the system states of Memristor-based Chua's oscillator have been stabilized using SMC and intelligent SMC. Given Fig. 4 \& 5, the hitting time has been decreased by using the intelligent SMC compared with SMC and chattering phenomenon has been reduced considerably.

Fig. 6 shows the tracking of a sinusoidal input by system output using intelligent SMC and SMC methods. In Fig. 7, the time response of $\mathrm{S}$ and $\mathrm{u}$ in tracking problem are shown.

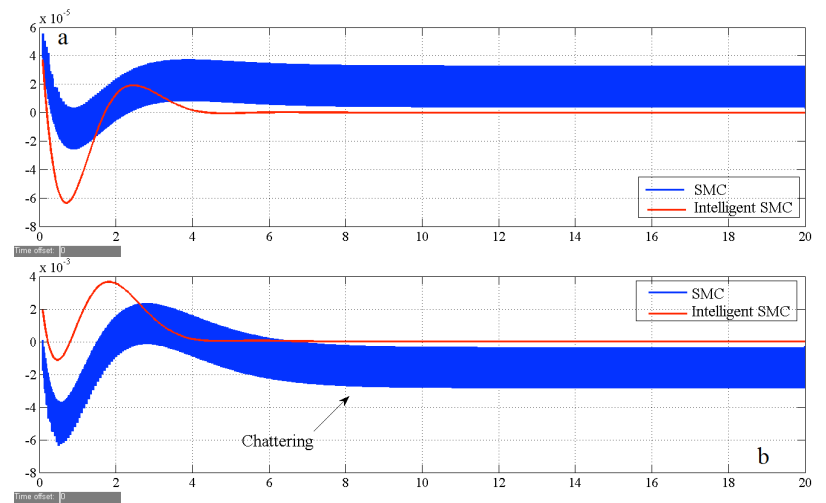

Fig. 5. The time response of $S$ and $u$ in stabilization problem using intelligent SMC and normal SMC, (a) $S$, (b) $u$.
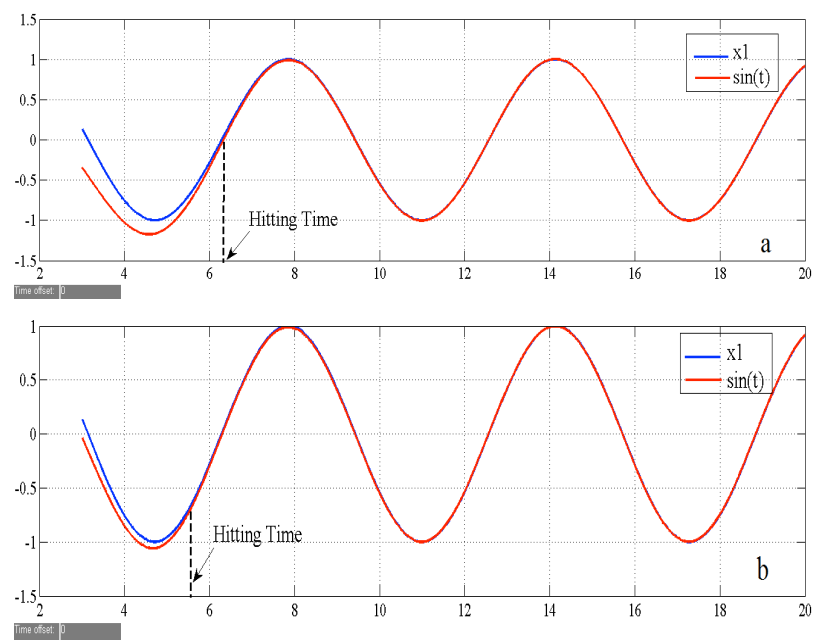

Fig. 6. The tracking of a sinusoidal input by system output using a) intelligent SMC and b) SMC.

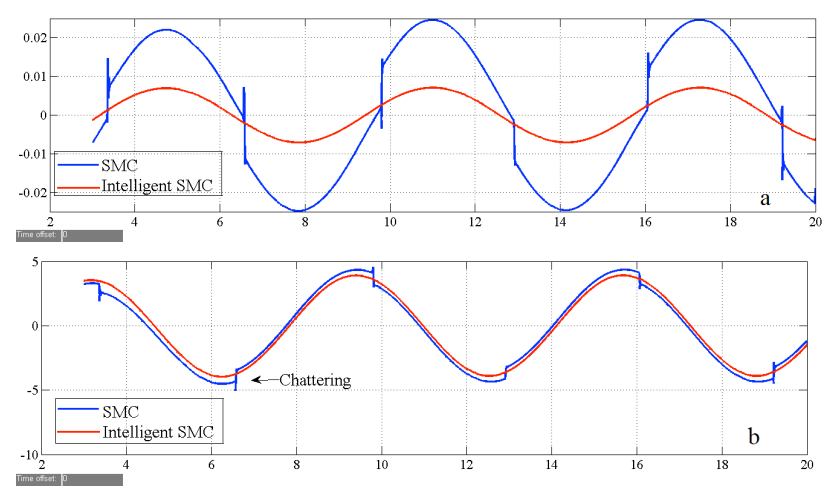

Fig. 7. The time response of $S$ and $u$ in tracking problem using intelligent SMC and SMC, (a) $S$, (b) $u$.

Given Fig.6 , in both intelligent SMC and SMC, the output of Memristor-based Chua's oscillator has tracked sinusoidal input as well.

From Fig. 6, we can see that hitting time of intelligent SMC is shorter than that of SMC. Additionally, as can be seen in Fig. 7, the chattering phenomenon problem in the SMC method is solved by GA optimization.

In Table 1, the comparison between hitting time values by two control methods is shown. The comparison of hitting 
time values shows that the hitting time is reduced in intelligent method.

\begin{tabular}{|c|c|c|c|c|c}
\cline { 2 - 6 } \multicolumn{1}{c|}{} & \multicolumn{5}{c}{ Hitting Time (sec) } \\
\cline { 2 - 6 } \multicolumn{1}{c|}{} & $\mathrm{X}_{1}$ & $\mathrm{X}_{2}$ & $\mathrm{X}_{3}$ & $\mathrm{X}_{4}$ & $\mathrm{~T}_{\text {Tracking }}$ \\
\hline SMC & 9.7 & 10.2 & 9.4 & 10.7 & 6.3 \\
\hline $\begin{array}{c}\text { Intelligent } \\
\text { SMC }\end{array}$ & 6.4 & 7.1 & 6.7 & 6.7 & 5.5 \\
\hline
\end{tabular}

Tab. 1. The comparison of hitting time values of control methods.

\section{Conclusion}

In this paper, chaos control problem in memristor- based oscillator using intelligent traditional SMC is investigated. Controlling chaos is performed to achieve two main goals of stabilization of system states and tracking the sinusoidal input by system output. Also the target of reducing the convergence time and avoiding chattering phenomenon is achieved by applying intelligent GA method. According to our results, it is shown that hitting time has decreased and chattering problem has been resolved in intelligent method.

\section{References}

1. H. Adelipoor and O.A. Babaei, Stability and tracking in the new chaotic system using bakstepping method, Journal of Basic and Applied Scientific Research, vol. 3(1), pp. 446-452 (2013).

2. R. Gholipour, A. Khosravi, and H. Mojallali, Suppression of chaotic behavior in Duffing-Holmes system using backstepping Controller Optimized by Unified Particle Swarm Optimization algorithm, International Journal of Engineering, Transactions B: Applications. vol. 26(11), pp. 1005-1015 (2013).

3. C. Guzelis and S. Sahin, "Chaotification" of real systems by dynamic state feedback, IEEE Antennas and Propagation Magazine, vol. 52(6), pp.222-233 (2010).

4. H. Ren and D. Liu, Nonlinear feedback control of chaos in permanent magnet synchronous motor adaptive, IEEE Transaction on Circuits and Systems II: Express Briefs, vol. 53(1), pp. 45-50 (2006).

5. C. Hu, H. Jiang, and Z. Teng, General impulsive control of chaotic systems based on a TS fuzzy model, fuzzy sets and systems, vol. 174, pp. 66-82 (2011).

6. Z. Jinfu, J. Murong, and Z. Xiaobing, Passivity-based adaptive chaos control of a new 3D chaotic system with uncertain Parameters, International Forum on Information Technology and application (IFITA), vol. 3, pp. 57-60 (2009).

7. L.O. Chua, Memristor-the missing circuit element, IEEE Transaction on Circuit Theory, vol. 18(5), pp. 507-519 (1971).

8. D.B. Strukov, G.S. Snider, D.R. Stewart, and R.S. Williams, The missing memristor found, Nature, vol. 453, pp. 80-83 (2008).

9. R. Williams, How we found the missing memristor, IEEE Spectrum, vol. 45(12), pp. 28-35 (2008).

10. G. Wang and A. Qi, Chaotic oscillator based on memristor and its circuit implementation, In 2011 Fourth International Workshop on Chaos-Fractals Theories and Applications, pp. 329-331 (2011).

11. A.H. Elsamman, A.H. Madian, and A.G. Radwan, The modified single input Op-amps memristor based oscillator, In Int. Conf. On Communications, Signal Processing, and their Applications, Sharjah, pp. 1-4 (2013).

12. A.H. Elsamman, A.H. Madian, and A.G. Radwan, Memristorbased oscillator using Deboo integrator,In Int. Conf. on Computer Engineering \& Systems, Cairo, pp. 103-107 (2012).

13. A. Talukdar, A.G. Radwan, and K.N. Salama, Generalized model for memristor-based Wien family oscillators, Microelectronics Journal, vol. 42, pp. 1032-1038 (2011).

14. M.A. Zidan, H. Omran, A.G. Radwan, and K.N. Salama, Memristor-based reactance-less oscillator, Electronics Letters, vol. 47(22), pp. 1220-1221 (2011).
15. H.H.C. Iu, V. Sreeram, A.L. Fitch, D.S. Yu, Controlling chaos in a memristor based circuit using a twin-T notch filter, IEEE Transaction on Circuits and Systems-I: Regular Papers, vol. 58(6), pp. 1337-1334 (2011).

16. Y. Song, S. Yi, and Y. Chang, Chaos control of a memristorbased Chua's oscillator via backstepping method, In Int. Conf. on Information Science and Technology, Nanjing, pp. 10811084 (2011).

17. S.S. Ge and C. Wang, Adaptive control of uncertain Chua's circuits, IEEE Transaction on Circuits I: Fundamentals Theory and Applications, vol. 47(9), pp. 1397-1404 (2009).

18. Y. Yongbin, J. Jin, F. Zhang, Q. Zhong, Impulsive modelling of memristor oscillator and its control, In Int. Conf. on Communications, Circuits and Systems, Chengdu, pp. 837-841 (2010).

19. C.C. Hwang, J.Y. Hsheh, and R.S. Lin, A linear continuous feedback control of Chua's circuit, Chaos, Solitons \& Fractals, vol. 8(9), pp. 1507-1515 (1997).

20. J.J.E. Slotine, W. Li, Applied nonlinear control, Prentice Hall, New Jersey (1991).

21. M.J. Mahmoodabadi, M. Taherkhorsandi, and A. Bagheri, Optimal robust sliding mode tracking control of a biped robot based on ingenious multi-objective PSO, An International Journal of Neurocomputing, vol. 124, pp. 194-209 (2014).

22. Y. Zhang, Sliding mode control with nonlinear disturbance observer based on genetic algorithm for rotary steering drilling stabilized platform, Research Journal of Applied Sciences, Engineering and Technology, vol. 6(17), pp. 3187-3192 (2013).

23. E. Köse, K. Abacı, H. Kızmaz, S. Aksoy, and M.A. Yalçın, Sliding mode control based on genetic algorithm for WSCC systems include of SVC, Elektronika IR Elektrotechnika, vol. 19(4), pp. 19-24 (2013).

24. F. Piltan, S. Siamak, M.A. Bairami, and I. Nazari, Gradient descent optimal chattering free sliding mode fuzzy control design: Lyapunov approach, International Journal of Advanced Science and Technology, vol. 45 (2012).

25. C.C. Wong, B.C. Huang, H.R. Lai, and J. Tamkang, Geneticbased sliding mode fuzzy controller design, Tamkang Journal of Science and Engineering, vol. 4(3), pp. 165-172 (2001).

26. M. Mitchell, An introduction to genetic algorithm, MIT Press, Cambridge, Massachusetts (1998).

27. G.Q. Zhong, Implementation of Chua's circuit with a cubic nonlinearity, IEEE Transactions on Circuits and Systems, vol. 41(12), pp. 934-941 (1994). 